\title{
Aplicação da Estratégia SPI-KM para Apoiar a Implementação do MPS.BR Níveis G e F em Pequenas e Médias Empresas do Rio de Janeiro
}

\author{
Gleison Santos, Mariano Montoni, Anne Elise Katsurayama, \\ Reinaldo Cabral, Sávio Figueiredo, Ana Candida Natali, Cristina Cerdeiral, \\ Jucele Vasconcellos, David Zanetti, Peter Lupo, Ana Regina Rocha \\ COPPE/UFRJ - Universidade Federal do Rio de Janeiro \\ Programa de Engenharia de Sistemas e Computação \\ Av. Horácio Macedo, 2030, Prédio do Centro de Tecnologia, Bloco H, Sala 319 \\ Caixa Postal 68511 - CEP 21941-914 - Rio de Janeiro, RJ \\ \{gleison, mmontoni, anneelisek, cabral, savio, anatali, \\ cerdeiral, jucele, zanetti, darocha\}@cos.ufrj.br
}

\begin{abstract}
During the last 5 years, Brazilian software industry and universities are working cooperatively to develop and disseminate the MPS Model, aiming to improve the quality of Brazilian software processes and products. In order to cope with difficulties Small and Medium-size Enterprises (SMEs) face during SPI implementation and to facilitate the dissemination of the MPS Model, we developed an approach, named SPI-KM, supported by a Process-centered Software Engineering Environment. This paper presents the results and lessons learned of the application of the SPI-KM approach in a group of 5 Brazilian SMEs that implemented the MPS Model.
\end{abstract}

Resumo. Nos últimos 5 anos, universidades e a indústria de software do Brasil têm trabalhado para desenvolver e disseminar o MPS.BR com o intuito de melhorar a qualidade dos produtos e processos de software brasileiros. Para melhor lidar com as dificuldades que pequenas e médias empresas enfrentam durante a implementação de programas de melhoria de processos de software e facilitar a disseminação do MPS.BR, foi desenvolvida uma abordagem, denominada SPI-KM, apoiada por um ambiente de engenharia de software centrado em processos. Este artigo apresenta os resultados e lições aprendidas pela aplicação desta estratégia num grupo de 5 empresas situadas no Rio de Janeiro durante a implementação dos níveis $G$ e F do MPS.BR.

\section{Introdução}

A melhoria de processos em pequenas empresas exige um tratamento diferenciado em virtude das restrições existentes em relação a recursos materiais e humanos. No entanto, para garantir sua sobrevivência, é essencial que estas empresas busquem superar estas dificuldades e aprimorar seu processo produtivo. Para apoiar este esforço para melhoria de processos em pequenas e médias empresas algumas abordagens têm sido propostas [Thiry et al. 2006]. O MPS.BR é um programa para Melhoria de Processo do Software Brasileiro que teve início em 2003, sob coordenação da Associação para Promoção da 
Excelência do Software Brasileiro (Softex), cujo principal objetivo é viabilizar a implantação dos princípios de Engenharia de Software de forma adequada ao contexto das empresas brasileiras em consonância com as principais abordagens internacionais para definição, avaliação e melhoria de processos de software [Softex 2006]. O Software Engineering Institute (SEI) também tem demonstrado interesse em pesquisas com foco em melhoria de processos em pequenas empresas [SEI 2006].

A COPPE/UFRJ tem trabalhado em consultoria para implementação de melhoria de processos de software nas organizações de software brasileiras por mais de duas décadas. A partir de 2003, a COPPE/UFRJ incluiu em sua estratégia a utilização do ambiente de apoio a processos de engenharia de software TABA configurado para cada empresa, fato determinante para a conquista dos níveis 2 e 3 do CMMI, além dos níveis F, E e D do MR-MPS em diferentes organizações [LENS 2007]. Desde 2003, a equipe da COPPE/UFRJ tem implantado processos de software em grupos de pequenas e médias empresas no Rio de Janeiro organizados pela Riosoft (que integra o Programa Softex). A partir de 2004 o foco destes grupos passou a ser a implantação de processos aderentes ao MR-MPS [Rocha et al. 2005].

Este artigo relata lições aprendidas a partir da experiência de implementação de melhoria de processos em um grupo de empresas visando a obtenção dos níveis $\mathrm{G}$ e $\mathrm{F}$ do MR-MPS entre 2006 e 2007. O grupo de empresas formado pela Riosoft em 2006 foi composto por 7 empresas, sendo que a COPPE/UFRJ atuou como Instituição Implementadora em 5 destas empresas e a Riosoft, em outras 2, que não serão abordadas neste trabalho. A seção 2 apresenta um breve histórico da experiência da COPPE/UFRJ na implementação de processos em pequenas e médias empresas do Rio de Janeiro e é discutida a estratégia de implementação adotada. A seção 3 apresenta as empresas. Na seção 4 são apresentadas as lições aprendidas e na seção 5 as considerações finais.

\section{Implementando Processos em Pequenas e Médias Empresas}

\subsection{Estratégia de Implementação da COPPE}

A implantação de processos de software em uma organização é fortemente baseada em conhecimento. Para a implantação de uma iniciativa de melhoria, é fundamental que os responsáveis por executar os processos sejam capacitados. Além disso, uma iniciativa de melhoria é normalmente considerada cara por muitas organizações, pois são necessários recursos significativos durante certo período de tempo. Desta forma, torna-se necessário desenvolver estratégias efetivas para implementar processos de software com sucesso. Com o objetivo de apoiar a implementação de iniciativas de implantação e melhoria de processos em organizações de software, foi desenvolvida, na COPPE/UFRJ, uma estratégia de implementação fortemente baseada em conhecimento [Santos et al. 2007] [Barreto et al. 2006]. Essa estratégia tem demonstrado sua viabilidade e seus benefícios através de muitas avaliações de processo realizadas nos últimos anos. Esta abordagem pode e está sendo aplicada em implementações tanto em empresas individuais quanto nas que participam de algum grupo de empresas. A Figura 1 apresenta esta abordagem, denominada SPI-KM.

Durante o passo inicial da estratégia ("Diagnóstico") é realizada a seleção do modelo de maturidade e o nível pretendido pela organização, além de verificação da 
cultura existente. Depois disso, a iniciativa de melhoria é planejada e um processo de desenvolvimento de software padrão é definido para a organização. Os profissionais são treinados nos processos a serem executados, tanto de forma teórica, como durante suas execuções através de "mentoring". Ao longo da execução dos processos, ocorre a aquisição e registro de conhecimento, além da coleta de recomendações de melhoria nos processos, que, posteriormente, são avaliadas e podem levar a mudanças na definição do processo. Por fim, é realizada a preparação da organização para a avaliação dos processos, onde todos os participantes tomam conhecimento do processo de avaliação a ser utilizado e de seus objetivos.

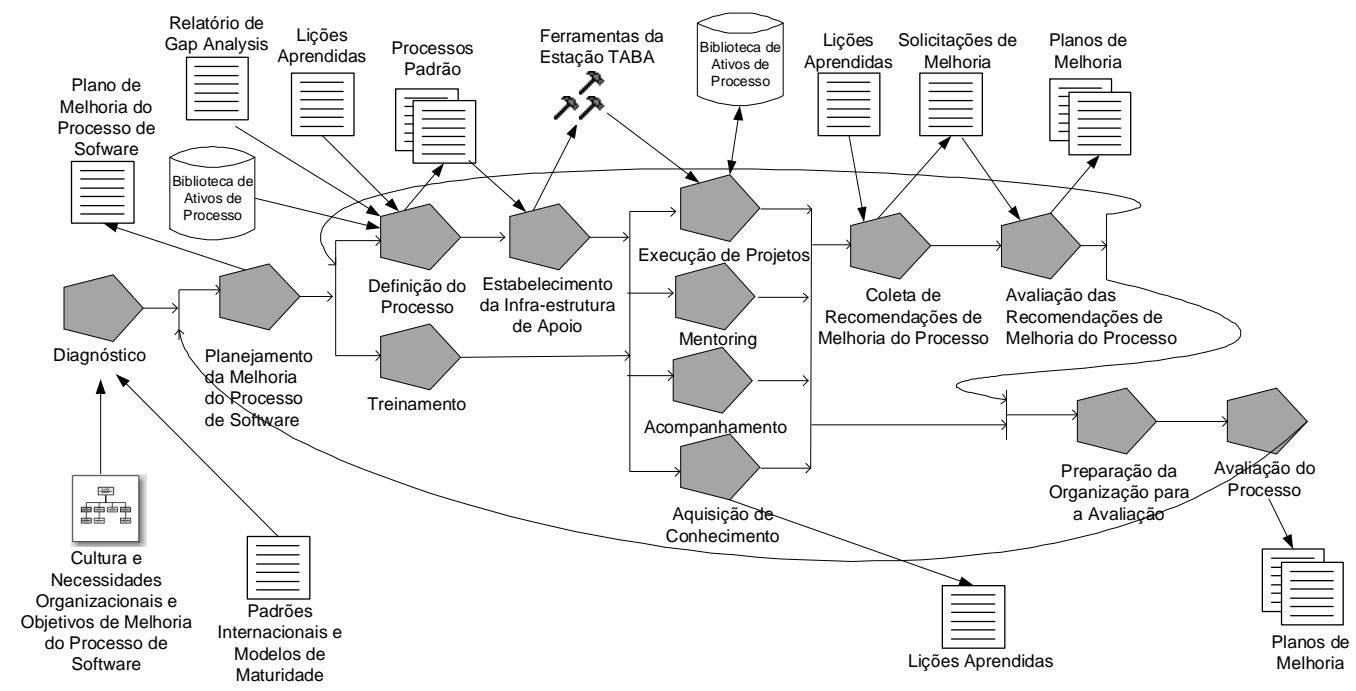

Figura 1. SPI-KM - Estratégia de Implementação de Processos da COPPE/UFRJ.

Alguns pontos chave desta estratégia são: (i) definição do processo: um conjunto de processos padrão para a organização é definido de acordo com o modelo de maturidade a ser adotado e elementos deste conjunto serão usados em todos os projetos de software da unidade organizacional envolvida na iniciativa de melhoria; (ii) treinamento: os membros da organização são treinados nas práticas que deverão ser utilizadas durante a execução dos processos e nas ferramentas que serão utilizadas nas atividades do processo definido; (iii) mentoring durante execução dos processos: os consultores da COPPE/UFRJ acompanham cada profissional envolvido na melhoria enquanto este realiza alguma atividade do processo; (iv) aquisição de conhecimento: envolve a aquisição de conhecimento relacionado às atividades do processo de forma a permitir que o conhecimento organizacional seja preservado e disseminado na organização; (v) apoio ferramental da Estação Taba: permite que as atividades sejam mais facilmente assimiladas e executadas; (vi) acompanhamento da implementação da melhoria de processos de software: ocorre regularmente, por exemplo, uma vez por semana, e envolve a discussão com os membros da organização responsáveis pelos detalhes da iniciativa de melhoria sobre diretivas importantes para manter a execução dos processos no rumo certo. Durante estas reuniões, estratégias de melhoria de processos são definidas para superar dificuldades inerentes à iniciativa de melhoria de processos que podem afetar 0 seu sucesso, como, por exemplo, resistência a mudanças por parte dos participantes.

Para apoiar a execução desta abordagem, são utilizados os ambientes, a infra- 
estrutura e as ferramentas da Estação TABA. A Estação TABA é capaz de gerar ambientes de desenvolvimento de software para apoiar atividades de gerência de projetos, melhoria da qualidade dos produtos e aumento da produtividade [Villela et al. 2004]. Durante os últimos anos, a Estação TABA evoluiu para apoiar atividades de gerência de conhecimento integradas aos processos de software com o objetivo de preservar o conhecimento organizacional e permitir a institucionalização de uma organização de software em contínuo aprendizado[Oliveira et al. 2004] [Villela et al. 2004]. Uma das principais ferramentas no que diz respeito à gerência do conhecimento é a ferramenta ACKNOWLEDGE, onde são registradas as diretrizes, lições aprendidas e demais itens de conhecimento, sempre associados a atividades do processo. Essa ferramenta está integrada a todas as ferramentas e ambientes da Estação TABA [Montoni et al. 2004].

\subsection{Lições Aprendidas Durante a Aplicação da Estratégia SPI-KM}

Lições aprendidas podem facilitar a implantação de processos de software em outras organizações no futuro e, assim, aumentar as chances de sucesso de novas iniciativas de melhoria. Um estudo feito pela COPPE/UFRJ [Santos et al. 2007] identificou problemas comuns à implementação de processos, independente da empresa ou estratégia utilizada. A partir deste estudo foram extraídas 5 lições aprendidas. Naquele momento, várias iniciativas de melhoria de processos sob nossa coordenação estavam em andamento. Após a conclusão destas iniciativas, pôde-se não apenas confirmar a validade e aplicabilidade de tais lições, mas também identificar uma nova e importante lição aprendida. Estas lições são apresentadas na Tabela 1.

Tabela 1. Lições aprendidas pela aplicação da estratégia SPI-KM

\begin{tabular}{|c|c|}
\hline Título & \\
\hline $\begin{array}{l}\text { Iniciativas de melhoria de } \\
\text { processos de software de- } \\
\text { vem efetivamente melhorar } \\
\text { os processos de software. }\end{array}$ & $\begin{array}{l}\text { O foco de um programa de melhoria de processos, que define as estraté- } \\
\text { gias, políticas, atividades e responsabilidades para condução da iniciativa, } \\
\text { deve estar vinculado aos objetivos de negócio da organização. }\end{array}$ \\
\hline $\begin{array}{l}\text { Você não terá sucesso sem } \\
\text { um líder. }\end{array}$ & $\begin{array}{l}\text { A melhoria de processos de software implica na adoção de novas práticas } \\
\text { pela organização. Muitas barreiras são encontradas durante as iniciativas } \\
\text { de melhoria, como por exemplo, políticas organizacionais, falta de apoio e } \\
\text { recursos, falta de conhecimento e pressões de cronograma [Niazi et al. } \\
\text { 2005]. Neste contexto, é muito importante contar com pessoas capazes de } \\
\text { motivar, sensibilizar e orientar os membros da organização na realização } \\
\text { de ações que permitam superar essas e outras dificuldades. }\end{array}$ \\
\hline $\begin{array}{l}\text { Comprometimento é } \\
\text { crucial. }\end{array}$ & $\begin{array}{l}\text { n o comprometimento de todos os níveis organizacionais, os objetivos } \\
\text { melhoria dificilmente serão alcançados [Abrahamsson et al. 2001]. } \\
\text { ssa forma, as pessoas envolvidas com as iniciativas de melhoria devem } \\
\text { ceber os benefícios derivados da melhoria e não somente seus custos. }\end{array}$ \\
\hline Sem cérebro, sem ganhos. & $\begin{array}{l}\text { ecimen- } \\
\text { elaciona- } \\
\text { nização. }\end{array}$ \\
\hline $\begin{array}{l}\text { A melhoria de processos de } \\
\text { software é facilitada pela } \\
\text { infra-estrutura de processos } \\
\text { de software. }\end{array}$ & $\begin{array}{l}\text { A maior parte das organizações com processos de software de baixa matu- } \\
\text { ridade não possui infra-estrutura adequada para as iniciativas de melhoria. } \\
\text { Zaharan (1998) define } 2 \text { tipos de infra-estrutura de apoio a atividades rela- } \\
\text { cionada a processos e para sustentar ações de melhoria: (i) organizacional } \\
\text { e gerencial, e (ii) técnica. }\end{array}$ \\
\hline $\begin{array}{l}\text { A descentralização do } \\
\text { conhecimento sobre a im- } \\
\text { plementação de processos } \\
\text { de software é fundamental }\end{array}$ & $\begin{array}{l}\text { A implementação de melhoria de processos é um projeto de longo prazo, } \\
\text { dessa forma, também pode ser afetada pela rotatividade de pessoal. Para } \\
\text { reduzir este e outros riscos relacionados às iniciativas de melhoria dentro } \\
\text { de uma organização é fundamental que o conhecimento sobre a implemen- }\end{array}$ \\
\hline
\end{tabular}


para sustentar as iniciativas tação seja descentralizado. Dessa forma, mais pessoas na empresa serão de melhoria de processos capazes de disseminar as melhorias e, também, o apoio e o comprometide software. mento dos colaboradores com os processos de software será maior.

\section{As Empresas do Grupo}

Nas cinco empresas do grupo Qualisoft 2006, todas localizadas na cidade do Rio de Janeiro, a estratégia de implementação utilizada foi a mesma, respeitando-se porém as características, experiência e maturidade de cada empresa.

A Organização \#1 é dedicada a fornecer soluções em sistemas para o mercado financeiro na área de investimentos. O objetivo de seu programa de melhoria de processos de software é aumentar a qualidade dos produtos, melhorar o controle dos projetos e diminuir os custos de produção e manutenção dos sistemas. A empresa já havia participado de outro esforço de melhoria que, apesar de não ter resultado em uma avaliação, trouxe maturidade para a organização em relação a conhecimento sobre processo e definição de objetivos de melhoria. $\mathrm{O}$ apoio da alta e média gerência, assim como dos colaboradores envolvidos nos projetos, é visível e sempre esteve presente. A empresa possui um grupo de processos atuante e qualificado para dar apoio aos projetos, que colaborou na definição de um processo bem adequado à realidade da empresa.

A Organização \#2 é especializada em prestação de serviços de informática, atuando na área de desenvolvimento de sistemas e processos customizados ao cliente e desenvolvimento de produtos próprios, para venda e uso interno. O objetivo do programa de melhoria de processos de software da empresa é aumentar a qualidade dos produtos e diminuir os custos de desenvolvimento. Os membros da organização envolvidos com o programa de melhoria bem como a alta gerência apóiam integralmente a iniciativa e observam resultados positivos.

A Organização \#3 fornece serviços em Business Intelligence, portais corporativos e soluções de sistemas de informação e gestão do conhecimento. A empresa estabeleceu como objetivo de qualidade o aprimoramento de seus processos e técnicas de desenvolvimento de software para aumentar a qualidade de seus produtos e diminuir o retrabalho existente. Os envolvidos e a alta gerência apóiam fortemente a iniciativa de melhoria e alegam que o programa, além de ser uma necessidade para aumentar a competitividade no mercado atual, representa uma questão de sobrevivência para a empresa. A organização trabalhou, paralelamente, na implantação da norma ISO 9001:2000.

A Organização \#4 é especializada no desenvolvimento e comercialização de pacotes de soluções, principalmente para os ramos da saúde, educação e finanças. O principal objetivo do programa de melhoria de processos de software da empresa é aumentar a qualidade dos produtos e diminuir o esforço de manutenção, devido à longa vida útil e adaptabilidade de seus produtos. Desde o início todos os envolvidos na implementação dos processos e a alta gerência da empresa apóiam a iniciativa e os resultados já são percebidos.

A Organização \#5 fornece serviços de desenvolvimento de software, integração de soluções, outsorcing de TI e modelagem de negócios. Tem como principal objetivo para o programa de melhoria de processos de software, melhorar a gerência dos projetos a fim de aumentar o controle de prazo, custo e esforço no desenvolvimento de software 
e diminuir o retrabalho. A empresa apóia o programa de melhoria. Existe uma participação ativa da direção e da gerência que demonstra acreditar nos benefícios da implementação dos processos do MR-MPS.

A Tabela 2 apresenta o nível desejado por cada empresa e o estágio atual de implantação dos processos. A Organização \#2 já tinha participado de treinamento teórico no Qualisoft 2005 e por isso não participou do treinamento neste grupo. A Organização \#4 optou por não ter treinamento teórico, no entanto, conceitos teóricos foram sendo introduzidos durante o acompanhamento nos projetos. A Organização \#1 e a Organização \#2 adaptaram a definição do processo segundo suas necessidades e isso aumentou o tempo da definição e configuração do Taba.

Tabela 2. Caracterização e Estágio Atual das Organizações

\begin{tabular}{|c|c|c|c|c|c|}
\hline & Organização \#1 & Organização \#2 & Organização \#3 & Organização \#4 & Organização \#5 \\
\hline Nível MR-MPS & $\mathbf{F}$ & $\mathbf{G}$ & $\mathbf{G}$ & $\mathbf{F}$ & G \\
\hline Início do Projeto & Jul/2006 & Jul/2006 & Jul/2006 & Jan/2007 & Jul/2006 \\
\hline $\begin{array}{l}\text { Definição do Processo } \\
\text { e Configuração TABA }\end{array}$ & Ago-Set/2006 & Ago-Nov/2006 & $\mathrm{Jul} / 2006$ & Jan/2007 & $\mathrm{Jul} / 2006$ \\
\hline Treinamento Teórico & Set/2006 & - & Jul/2006 & - & Ago/2006 \\
\hline Início da Implantação & Set/2006 & Nov/2006 & Ago/2006 & Jan/2007 & Ago/2006 \\
\hline $100 \%$ da Implantação & Jul/2007 & Mai/2007 & Jan/2007 & Jun/2007 & Jun/2007 \\
\hline $\begin{array}{l}\text { Avaliação Inicial } \\
\text { (MA-MPS) }\end{array}$ & Ago/2007 & Mai/2007 & Abr $/ 2007$ & Ago-Set/2007 & Set/2007 \\
\hline $\begin{array}{l}\text { Avaliação Final } \\
\text { (MA-MPS) }\end{array}$ & Out/2007 & Jun/2007 & Mai/2007 & Out/2007 & Set/2007 \\
\hline $\begin{array}{l}\text { Tempo total para a } \\
\text { avaliação final a partir } \\
\text { do início da implemen- } \\
\text { tação na empresa }\end{array}$ & 15 meses & 12 meses & 11 meses & 10 meses & 14 meses \\
\hline
\end{tabular}

\section{Lições Aprendidas}

Durante a implementação do programa de melhoria nestas empresas foram utilizadas as lições aprendidas durante a aplicação da abordagem SPI-KM (apresentada na seção 2) de forma a reduzir os riscos inerentes às iniciativas de melhoria de processos de software conduzidas sob nossa coordenação. Durante a execução destas iniciativas de melhoria, utilizando a estratégia SPI-KM, neste grupo de empresas também foi possível coletar outro conjunto de lições aprendidas. Estes dois conjuntos de lições não são concorrentes, mas sim complementares. A Tabela 3 apresenta lições aprendidas que podem ser úteis na definição de um plano estratégico para melhoria de processos de software.

Tabela 3. Lições aprendidas

\begin{tabular}{|l|l|}
\hline$\#$ & \multicolumn{1}{|c|}{ Lição aprendida } \\
\hline $\mathbf{1}$ & $\begin{array}{l}\text { A presença constante dos consultores favorece a conscientização das vantagens e dos benefícios da } \\
\text { implantação do programa de melhoria na organização. Entretanto isso só ocorre se os consultores } \\
\text { também atuam visando a melhoria efetiva da organização e não apenas o sucesso da avaliação. }\end{array}$ \\
\hline $\mathbf{2}$ & $\begin{array}{l}\text { Empresas que nunca seguiram um processo têm muita dificuldade para definir um, portanto, é impor- } \\
\text { tante que a consultoria defina uma versão inicial do processo a ser executado. Com a utilização deste } \\
\text { processo em um projeto a empresa passa a ter o conhecimento necessário para apoiar a definição de } \\
\text { uma nova versão mais adequada à sua realidade. Além disso, as organizações aprendem bastante e } \\
\text { ficam mais comprometidas com o programa de melhoria e com a aderência do processo aos projetos. }\end{array}$ \\
\hline $\mathbf{3}$ & O primeiro projeto a utilizar o processo definido normalmente apresenta dificuldades, pois a equipe \\
\hline
\end{tabular}


está se adaptando às novas atividades e ferramentas que exigem uma mudança na forma como os projetos são conduzidos. Em alguns casos este projeto é encarado como um piloto para a implantação do processo e não é incluído no conjunto de projetos a fazerem parte da avaliação oficial do MPS.BR

Um importante ponto a ser considerado é a obtenção de comprometimento ao longo do projeto, pois

4 nem sempre as empresas estão habituadas a registrar este comprometimento tanto interna como ex-

ternamente. Pode haver resistências, principalmente do cliente, em relação ao estabelecimento formal de comprometimento.

Apoio ferramental adequado facilita o treinamento, desenvolvimento e institucionalização dos processos, ajudando a reduzir o tempo de implementação dos processos. Quando a organização já utiliza ferramentas adequadas para apoiar as atividades do processo, a resistência a mudanças diminui, fa-

5 zendo com que a implementação não tenha impacto na curva de aprendizado de novas ferramentas.

5 No que tange o uso da Estação TABA, ainda que no futuro a empresa opte por outras ferramentas que considere mais adequadas à sua realidade, é importante o contato com a Estação Taba para sedimentar os novos conceitos e ajudar na definição dos requisitos para outras ferramentas que se deseje utilizar.

A estratégia baseada na implantação gradativa de processos aderentes ao MR-MPS é adequada, pois

6 a organização percebe, aos poucos, os benefícios de disciplinar o desenvolvimento com base em processos.

Empresas pequenas que pretendem implantar o nível G do MR-MPS, geralmente possuem recursos financeiros limitados, ocasionando problemas para o programa de melhoria. Quanto mais rápido os

7 processos forem institucionalizados nestas empresas, menores serão os riscos de estes não serem seguidos, pois nestas empresas, geralmente, surgem problemas que podem impactar no andamento da implementação. Partir de uma versão inicial e adaptá-la à empresa acelera esta implantação e institucionalização.

Quando os membros responsáveis pela execução dos processos na organização possuem conhecimen-

8 to em engenharia de software há uma demanda menor por acompanhamento da consultoria e a implementação dos processos é agilizada, reduzindo, também os custos de implementação.

Com o maior controle, tanto gerencial como dos requisitos, que inicia desde o nível $\mathrm{G}$, as empresas

9 conseguem negociar novos prazos e custos com os clientes, principalmente quando ocorrem mudanças de requisitos, demonstrando quantitativamente o impacto das mudanças para o projeto.

\section{Considerações Finais}

Este artigo discutiu a implementação do MR-MPS num grupo de pequenas e médias empresas do Rio de Janeiro. Foram apresentadas as principais características destas empresas, incluindo seus objetivos para o programa de melhoria de processos, caracterização e estágio da implementação em cada uma das empresas. Além disso, foram apresentadas as lições aprendidas a partir da experiência de implementação de melhoria de processos visando a obtenção dos níveis G e F do MR-MPS.. Todas as empresas foram avaliadas e obtiveram os níveis pretendidos, o que comprova o sucesso da abordagem.

Atualmente a Organização \#4 definiu uma nova versão dos processos para atender toda a organização, englobando projetos de customização, integração e migração, além dos projetos de desenvolvimento. A Organização \#3 definiu processos adequados ao Nível E do MPS visando uma avaliação ainda em 2008. Um projeto já foi iniciado seguindo este novo processo, os grupos de processos foram definidos com recursos da organização com apoio da consultoria. A Organização \#5 continua usando os processos em projetos grandes e estuda adaptações para projetos pequenos. Além disso, está começando os preparativos para uma avaliação no Nível E do MPS em 2008. A Organização \#1, também, continua seu esforço de melhoria em direção ao nível E do MPS.

As lições aprendidas relatadas neste trabalho são importantes ativos que atuam 
na retroalimentação e refinamento da estratégia adotada pela COPPE/UFRJ. Da mesma forma, espera-se que estes ativos também possam contribuir para as estratégias de outras Instituições Implementadoras MPS ou de empresas que queiram realizar programas de melhoria de processos de software.

\section{Agradecimentos}

Os autores agradecem a todos os colaboradores das empresas do grupo, a Márcio Pecegueiro do Amaral e à equipe de desenvolvimento da Estação TABA.

\section{Referências}

Abrahamsson, P. (2001), Commitment Development in Software Process Improvement: Critical Misconceptions, In: Proc. of the 23rd Int. Conf. on Sof. Eng., pp. 71-80.

Barreto, A., Montoni, M., Santos, G., Rocha, A. R. (2006) "Gerência de Conhecimento como Apoio para Implantação de Processos de Software", II Workshop de Implementadores MPS.BR (W2-MPS.BR), Rio de Janeiro, Brasil.

LENS - Laboratório de Engenharia de Software da COPPE/UFRJ (2007) $<$ http://lens.cos.ufrj.br/>.

Montoni, M., Santos, G., Villela, K., Miranda, R., Rocha, A.R., Travassos, G.H., Figueiredo, S., Mafra, S.: Knowledge Management in an Enterprise-Oriented Software Development Environment. In.: Lecture Notes of Computer Science (LNCS), ISBN 3-540-24088-8, pp. 117-128, presented at the 5th Int. Conf of Practical Aspects of Knowledge Management, Vienna, Austria, (2004)

Niazi, M., Wilson, D., Zowghi, D. (2005), "A framework for assisting the design of effective software process improvement implementation strategies", In: J. of Systems and Software, v.78, n.2, pp. 204-222.

Oliveira, K, Zlot, F., Rocha, A. R., Travassos, G., Galotta, C., Menezes, C. (2004), Domain Oriented Software Development Environment, Journal of Systems and Software, vol 72/2 pp 145-161

Rocha, A. R., Montoni, M., Santos, G., Mafra, S., Figueiredo, S., Bessa, A., Mian, P. (2005) "Estação TABA: Uma Infra-estrutura para Implantação do Modelo de Referência para Melhoria de Processo de Software", IV Simpósio Brasileiro de Qualidade de Software, SBQS'05, Porto Alegre, Brasil.

Santos, G., Montoni, M., Figueiredo, S., Rocha, A. R. (2007) "SPI-KM - Lessons Learned from Applying a Software Process Improvement Strategy Supported by Knowledge Management", $8^{\text {th }}$ International Conference on Product Focused Software Process Improvement (PROFES'2007), Latvia.

SEI - Software Engineering Institute (2006) "Improving Processes in Small Settings (IPSS) - A White Paper", The International Process Research Consortium (IPRC), Software Engineering Institute, Pittsburgh.

Softex, MPS.BR - Melhoria de Processo do Software Brasileiro, Guia Geral (v. 1.1) . Sociedade Softex, Brasil. (2006)

Thiry, M., von Wangenheim, C. G., Zoucas, A., Pickler, K., "Uma Abordagem para a Modelagem Colaborativa de Processos de Software em Micro e Pequenas Empresas", V SBQS'2006, Vila Velha, ES, Brasil.

Villela, K., Santos, G., Montoni, M., Berger, P., Figueiredo, S, M., Mafra, S, N., Rocha, A, R, C., Travassos, G, H. (2004) "Definição de Processos em Ambientes de 
Desenvolvimento de Software Orientados a Organização", III SBQS, Brasília-DF.

Ye, Y. (2006), "Supporting software development as knowledge-intensive and collaborative activity", Proceedings of the 2006 International Workshop on Interdisciplinary Software Engineering Research, pp. 15-21, Changai, China

Zaharan, S. (1998), "Software Process Improvement - Practical Guidelines for Business Success", Addison-Wesley. 